\title{
THE SILVERMAN NECESSARY CONDITION FOR MULTIPLE INTEGRALS IN THE CALCULUS OF VARIATIONS
}

\author{
J. ERNEST WILKINS, JR.
}

\begin{abstract}
A necessary condition for a weak relative minimum of a multiple integral in the calculus of variations, recently derived by Silverman, is shown to be a consequence of (and hence equivalent to) the well-known Legendre-Hadamard necessary condition in the special cases of a double, triple or quadruple integral.
\end{abstract}

The Legendre-Hadamard condition $[1$, p. 11] is that

$$
Q(\xi, \lambda) \equiv f_{i j}^{\alpha \beta} \lambda_{\alpha} \lambda_{\beta} \xi^{i} \xi^{j} \geqq 0
$$

for all $\xi=\left(\xi^{1}, \xi^{2}, \cdots, \xi^{N}\right)$ and $\lambda=\left(\lambda_{1}, \lambda_{2}, \cdots, \lambda_{v}\right)$. (We employ the usual summation convention involving repeated indices. Greek indices range from 1 to $v$ and Latin indices from 1 to $N$. The relation of $f_{i j}^{\alpha \beta}$ to the integrand of a multiple integral (with $v$ independent variables), whose minimum value is sought, is known [1, p. 11] but immaterial to this paper.) The Silverman condition [2] is that

$$
Q^{\prime}(\Xi, \Lambda)=f_{i j}^{\alpha \beta}\left(\lambda_{\alpha}^{\gamma} \lambda_{\beta}^{\gamma} \xi_{\varepsilon}^{i} \xi_{\varepsilon}^{j}+\lambda_{\alpha}^{\gamma} \lambda_{\beta}^{\varepsilon} \xi_{\gamma}^{i} \xi_{\varepsilon}^{j}+\lambda_{\alpha}^{\gamma} \lambda_{\beta}^{\varepsilon} \xi_{\varepsilon}^{i} \xi_{\gamma}^{j}\right) \geqq 0
$$

for all $\Xi=\left(\xi_{\varepsilon}^{i}\right)$ and $\Lambda=\left(\lambda_{\alpha}^{\gamma}\right)$.

Silverman showed that his condition implies the Legendre-Hadamard condition, but left the converse open. It is, however, an immediate consequence of the easily verified identity,

$$
\begin{aligned}
2 Q^{\prime}(\Xi, \Lambda)= & 2(4-v) \sum_{\gamma} Q\left(\xi_{\gamma}, \lambda^{\gamma}\right) \\
& +\sum_{\gamma<\varepsilon}\left\{Q\left(\xi_{\gamma}+\xi_{\varepsilon}, \lambda^{\gamma}+\lambda^{\varepsilon}\right)+Q\left(\xi_{\gamma}-\xi_{\varepsilon}, \lambda^{\gamma}-\lambda^{\varepsilon}\right)\right\},
\end{aligned}
$$

in which $\xi_{\gamma}=\left(\xi_{\gamma}^{1}, \xi_{\gamma}^{2}, \cdots, \xi_{\gamma}^{N}\right), \lambda^{\gamma}=\left(\lambda_{1}^{\gamma}, \lambda_{2}^{\gamma}, \cdots, \lambda_{v}^{\gamma}\right)$, that the converse is true, and hence the Silverman and Legendre-Hadamard conditions are equivalent, when $v \leqq 4$. The situation when $v \geqq 5$ remains open.

Received by the editors September 13, 1973.

AMS (MOS) subject classifications (1970). Primary 49B20; Secondary 15A63, 26A86.

Key words and phrases. Legendre-Hadamard necessary condition.

(c) American Mathematical Society 1974 


\section{REFERENCES}

1. C. B. Morrey, Jr., Multiple integrals in the calculus of variations, Die Grundlehren der math. Wissenschaften, Band 130, Springer-Verlag, New York, 1966. MR 34 \#2380.

2. E. Silverman, A necessary condition in the calculus of variations, Proc. Amer. Math. Soc. 37 (1973), 462-464.

Physics Department, Howard University, Washington, D.C. 20001 